\title{
Escorpionismo em crianças e adolescentes: dados de uma unidade sentinela
}

\author{
Scorpionism in children and adolescents: data from a sentinel unit \\ Escorpionismo en niños y adolescentes: datos de una unidad centinela
}

Recebido: 18/01/2021 | Revisado: 23/01/2021 | Aceito: 24/01/2021 | Publicado: 30/01/2021

\author{
Patrícia Junglos \\ ORCID: https://orcid.org/ 0000-0001-9387-947X \\ Universidade Estadual de Maringá, Brasil \\ E-mail: pattyjunglos@ hotmail.com \\ Bianca Machado Cruz Shibukawa \\ ORCID: https://orcid.org/0000-0002-7739-7881 \\ Universidade Estadual de Maringá, Brasil \\ E-mail: bih.cruuz@gmail.com \\ Fernanda Ferreira Evangelista \\ ORCID: https://orcid.org/0000-0001-9576-3075 \\ Universidade Estadual de Maringá, Brasil \\ E-mail: fer.evangelista@ hotmail.com \\ Maria de Fátima Garcia Lopes Merino \\ ORCID: https://orcid.org/0000-0001-6483-7625 \\ Universidade Estadual de Maringá, Brasil \\ E-mail: fatimamerino@gmail.com \\ Ieda Harumi Higarashi \\ ORCID: https://orcid.org/0000-0002-4205-6841 \\ Universidade Estadual de Maringá, Brasil \\ E-mail: ieda1618@gmail.com \\ Magda Lúcia Félix de Oliveira \\ ORCID: https://orcid.org/0000-0003-4095-9382 \\ Universidade Estadual de Maringá, Brasil \\ E-mail: mlfoliveira@uem.br \\ Marcela Demitto Furtado \\ ORCID: https://orcid.org/0000-0002-0939-9615 \\ Universidade Estadual de Maringá, Brasil \\ E-mail: mar_demitto@hotmail.com
}

\begin{abstract}
Resumo
Objetivo: analisar o perfil epidemiológico de acidentes escorpiônicos em crianças e adolescentes em um centro de assistência toxicológica e apresentar o comportamento temporal das ocorrências. Método: estudo transversal, retrospectivo, realizado no Centro de Controle de Intoxicações no Noroeste do Paraná. A amostra foi constituída por fichas de notificação de crianças e adolescentes atendidas no centro de referência de 2014 a 2018. Foram analisadas 78 fichas por meio de estatística descritiva e para análise de tendência foi utilizado o teste de Mann-Kendall. Resultados: A maioria dos casos acometeu crianças de até cinco anos e do sexo masculino; grande parte foi classificada como leve, e dois evoluíram à óbito. Houve crescimento de casos no período. Conclusão: há incidência maior em crianças menores de cinco anos, e também uma crescente no número de casos ao longo do período estudado, o que configura um sério problema de saúde pública no município estudado e região.
\end{abstract}

Palavras-chave: Saúde da criança; Saúde do adolescente; Picadas de escorpião; Envenenamento; Epidemiologia.

\begin{abstract}
Objective: to analyze the epidemiological profile of scorpionic accidents in children and adolescents in a toxicological assistance center and to present the temporal behavior of the occurrences. Methods: cross-sectional, retrospective study, carried out at the Poison Control Center in Northwest Paraná. The sample consisted of notification forms for children and adolescents attended at the reference center from 2014 to 2018.78 forms were analyzed using descriptive statistics and the Mann-Kendall test was used for trend analysis. Results: The majority of cases involved children up to five years old, male; most were classified as mild, and two evolved to death. There was an increase in cases in the period. Conclusion: there is a higher incidence in children under five years of age, and also an increasing number of cases over the period studied, which represents a serious public health problem in the studied municipality and region. Keywords: Child health; Adolescent health; Scorpion stings; Poisoning; Epidemiology.
\end{abstract}

\section{Resumen}

Objetivo: analizar el perfil epidemiológico de los accidentes escorpiónicos en niños y adolescentes en un centro de asistencia toxicológica y presentar el comportamiento temporal de los sucesos. Métodos: estudio transversal, 
retrospectivo, realizado en el Centro de Control de Intoxicaciones del Noroeste de Paraná. La muestra consistió en formularios de notificación para niños y adolescentes atendidos en el centro de referencia entre 2014 y 2018 .Se analizaron 78 formularios mediante estadística descriptiva y se utilizó la prueba de Mann-Kendall para el análisis de tendencias. Resultados: La mayoría de los casos involucraron a niños de hasta cinco años, varones; la mayoría se clasificó como leve y dos evolucionaron hasta la muerte. Hubo un aumento de casos en el período. Conclusión: existe una mayor incidencia en menores de cinco años, y también un número creciente de casos durante el período estudiado, lo que representa un grave problema de salud pública en el municipio y región estudiados.

Palabras clave: Salud del niño; Salud del adolescente; Picaduras de escorpión; Intoxication; Epidemiología.

\section{Introdução}

Os acidentes com escorpiões e outros animais peçonhentos são uma emergência clínica e representam um sério problema de saúde pública com grande relevância no público pediátrico (Araújo et al., 2017).

O escorpionismo é um quadro de envenenamento causado pela inoculação de toxinas que determina quais alterações locais e sistêmicas vão acometer o indivíduo (Lisboa et al., 2020). A picada de escorpião produz uma estimulação de terminações nervosas com quadro local de dor intensa, edema, eritema e sudorese localizada. No quadro sistêmico observa-se sudorese profusa, agitação psicomotora, tremores, náuseas, vômitos, sialorreia, hipertensão ou hipotensão arterial, arritmia, edema agudo de pulmão e choque. Este quadro é mais observado nas crianças com evolução de gravidade (Ministério da Saúde, 2019).

Alguns fatores influenciam o quadro clínico da vítima, como extremos de idade; massa corporal; quantidade de peçonha inoculada; espécie e tamanho do animal; local da picada, condição da vítima antes da picada; predisposição alérgica e também o atraso no atendimento ${ }^{4}$. Sabendo disso, podemos salientar que apesar das manifestações clínicas estarem relacionadas à quantidade de peçonha inoculada, geralmente os adultos apresentam manifestações locais e as crianças $(<14$ anos) apresentam manifestações sistêmicas (Ministério da Saúde, 2019).

No Brasil existem 160 espécies de escorpiões catalogadas e três destas, são responsáveis por envenenamento grave e até casos fatais na região Sul, Sudeste, Centro-Oeste e Nordeste, são elas: T. Bahiensis (marrom), T. Serrulatus (amarelo) considerada a espécie mais perigosa para seres humanos - e T. Stigmurus. Estes artrópodes podem ser encontrados em habitações humanas, construções, terrenos baldios e também em locais onde existam insetos e acúmulo de matéria orgânica, áreas ao qual favorecem condições para alimento, abrigo e clima adequado para sobrevivência. Normalmente estes animais costumam ter hábitos noturnos e procuram esconderijos em locais escuros e úmidos durante o dia. Existe uma predominância dos acidentes em meses mais quentes e chuvosos devido à maior atividade relacionada à reprodução (Ministério da Saúde, 2009). Uma pesquisa revela aumento no número de notificações com estes artrópodes nos meses de outubro, novembro e dezembro (Feitosa et al., 2020).

O perfil epidemiológico dos acidentes com escorpiões no Brasil revela um aumento gradativo do ano de 2000 a 2017. Segundo o último boletim do Ministério da Saúde (MS) de 2017, foram notificados 124.077, com uma incidência de 59,7/100.000 habitantes e destes, 143 óbitos no total (Ministério da Saúde, 2019a; Ministério da Saúde, 2019b).

No Estado do Paraná estes acidentes assumem relevância, já que se verifica um aumento na frequência com que eles ocorrem. Nos anos de 2000 a 2019 foram notificados 18,076 casos, e somente no ano de 2019, essas notificações foram de 3.309. Neste mesmo ano houve uma incidência de 92,6/10.000 habitantes (Ministério da Saúde, 2020).

Diante disso, estudos que buscam analisar os casos de escorpionísmo em crianças e adolescentes são de grande relevância, pois poderão contribuir para o planejamento das ações de saúde e direcionar uma assistência adequada a este grupo, que é um dos mais vulneráveis, e assim reduzir a morbimortalidade por um possível agravo. Além disso, soma-se a justificativa para a realização desta pesquisa a escassez de trabalhos científicos atualizados sobre o tema na área de pediatria (Araújo et al., 2017; Feitosa et al., 2020; Lisboa et al., 2020; Ministério da Saúde, 2019a). 
Nesse sentido, o objetivo deste estudo foi analisar o perfil epidemiológico dos acidentes com escorpiões em crianças e adolescentes notificados a um Centro de Controle de Intoxicações, no período de janeiro de 2014 a dezembro de 2018 e apresentar o comportamento temporal das ocorrências.

\section{Metodologia}

Trata-se de um estudo transversal e retrospectivo com abordagem quantitativa (Pereira et al., 2018) e documental, realizado no Centro de Controle de Intoxicações de um Hospital Universitário da Região Noroeste do Paraná (CCI/HUM), que funciona 24 horas por dia, todos os dias da semana e conta com profissionais e estudantes da área da saúde. O mesmo atende todos os municípios da $15^{\circ}$ Regional de Saúde, além de outros Municípios, quando solicitado.

O Centro desenvolve um trabalho de esclarecimento à população e auxilia os profissionais de saúde a prestarem os primeiros socorros e a prescreverem o tratamento adequado em casos de acidente escorpiônico, além de fornecer atendimento presencial.

A população do estudo foi constituída por crianças e adolescentes ( 0 a 19 anos) que tiveram os casos notificados para o CCI/HUM durante o período de janeiro de 2014 a dezembro de 2018 e que ficaram internadas por um período igual ou superior a 12 horas, conforme critério utilizado pelo setor. Os critérios de exclusão foram: pacientes com idade igual ou maior que 20 anos e que ficaram em observação por um tempo menor que 12 horas.

A coleta de dados foi realizada nos meses de junho e julho de 2019 no CCI/HUM, por meio das fichas de notificações das crianças e adolescentes vítimas de acidentes por escorpiões, as quais totalizaram 78. As informações foram extraídas destas fichas, que são utilizadas pelo setor na identificação e notificação dos casos, para uma tabela elaborada pela pesquisadora no programa da Microsoft Office Excel 2010.

A análise dos dados se deu por meio da descrição dos dados referentes às variáveis: idade, sexo, zona de ocorrência do acidente e sua localidade, tempo decorrido entre exposição e atendimento, evolução do caso, classificação do envenenamento, agente, região anatômica da picada, alteração local, alteração sistêmica, alterações de sinais vitais e as alterações de exames complementares.

A princípio, foi realizada uma análise descritiva dos resultados para a obtenção de gráficos e tabelas de frequência, com o intuito de caracterizar as ocorrências. Para descrição dos resultados foram utilizadas a frequência absoluta e a porcentagem para as variáveis categóricas. A frequência absoluta $\left(n_{i}\right)$ é dada pelo número de vezes em que uma determinada variável assume um determinado valor/categoria em questão. A porcentagem $\left(p_{i}\right)$ é o resultado da razão entre a frequência absoluta e o tamanho da amostra, multiplicado por 100, isto é, $100 . \frac{n_{i}}{n} \%$.

Para verificar a existência de tendência nas séries mensais de casos, foi utilizado o teste não paramétrico de MannKendall que avalia a presença de tendência em uma série temporal, seja linear ou não linear (Machiwall \& Jha, 2012). Ainda, foi obtida a estatística $\tau$ de Mann-Kendall, cujo sinal indica se a tendência é crescente $(\tau>0)$ ou decrescente $(\tau<0)$.

Todas as análises foram realizadas com o auxílio do ambiente estatístico $\mathrm{R}(R$ Development Core Team), versão 3.5 ( $\mathrm{R}$ Development Core Team, 2015).

A pesquisa foi aprovada pelo Comitê Permanente de Ética em Pesquisa (COPEP) da Universidade Estadual de Maringá (UEM), conforme parecer n 3.540.336, e respeitou todos os princípios éticos e legais da Resolução 466/12, do conselho Nacional de Saúde. Como foram utilizados dados secundários, houve a dispensa do Termo de Consentimento Livre e Esclarecido. 


\section{Resultados}

Foram analisados 78 casos de acidentes escorpiônicos entre crianças e adolescentes no período de janeiro de 2014 a dezembro de 2018. Grande parte dos acidentes ocorreu em crianças com até cinco anos de idade (47,44\%), 28,21\% entre a idade de 6 e 10 anos, $14,10 \%$ entre 11 e 15 anos, e 10,26\% entre 16 e 19 anos (Tabela 1).

Tabela 1. Características sociodemográficas de crianças e adolescentes notificados no CCI/HUM por acidentes escorpiônicos, entre janeiro de 2014 a dezembro de 2018. Paraná, Brasil, 2019.

\begin{tabular}{lcc}
\hline Variável & Frequência absoluta & \% \\
\hline Idade (anos) & 37 & 47,44 \\
$\leq 5$ & 22 & 28,21 \\
6 a 10 & 11 & 14,10 \\
11 a 15 & 8 & 10,26 \\
16 a 19 & & \\
Sexo & 37 & 47,44 \\
Feminino & 41 & 52,56 \\
Masculino & & \\
Zona de moradia & 68 & 87,18 \\
Urbana & 6 & 7,69 \\
Rural & 4 & 5,13 \\
Não informado & & \\
Local da ocorrência & 70 & 59,74 \\
Residência & 4 & 5,13 \\
Outros* & 4 & 5,13 \\
Não informado & & \\
\hline
\end{tabular}

*Local de trabalho; Creche/Escola.

Fonte: Autoras.

Destacou-se também que, a maioria dos acidentes ocorreu entre crianças e adolescentes do sexo masculino (52,56\%), na zona urbana $(87,18 \%)$ e cujo local do evento foi a própria residência $(89,74 \%)$.

Em dois terços dos casos avaliados o agente foi o TityusSerrulatus $(66,67 \%)$ e em quase um quarto deles, o agente não foi identificado $(24,35 \%)$. Com relação ao tempo decorrido entre a exposição e o atendimento, nota-se que mais de $60 \%$ foi inferior há uma hora e que as regiões anatômicas da picada mais frequentes foram os membros inferiores $(51,28 \%)$ ou superiores $(35,90 \%)$ respectivamente (Tabela 2$)$.

Tabela 2. Características epidemiológicas de crianças e adolescentes notificados no CCI/HUM por acidentes escorpiônicos, entre janeiro de 2014 a dezembro de 2018. Paraná, Brasil, 2019. 
Research, Society and Development, v. 10, n. 1, e54610112079, 2021

(CC BY 4.0) | ISSN 2525-3409 | DOI: http://dx.doi.org/10.33448/rsd-v10i1.12079

Variável

Frequência absoluta

$\%$

Agente

TityusSerrulatus

52

$66,67 \%$

Bothriurus

6

$7,69 \%$

TityusBahienses

$1,28 \%$

Não identificado

$24,36 \%$

Tempo entre exposição e $1^{\circ}$ atendimento

Até 1 hora

$60,26 \%$

Até 2 horas

9

$11,54 \%$

Até 5 horas

$14,10 \%$

Mais de 5 horas

Não informado

$5,13 \%$

$8,97 \%$

Região anatômica da picada*

Membros inferiores

$51,28 \%$

Membros superiores

Tórax

Outros**

$10,25 \%$

Não informado

Alteração local*

Dor

Hiperemia

Eritema

Edema

Parestesia

Outros

Não informado

Alteração sistêmica*

Vômito

Taquicardia

Náusea

Agitação psicomotora

$11,54 \%$

Sonolência

Sudorese

Sialorréia

$5,13 \%$

Tremores

$5,13 \%$

Cefaléia

$5,13 \%$

Outros

Não informado

$5,13 \%$

Envenenamento

Leve

Moderado

Grave

Evolução 


$\begin{array}{llr}\text { Evasão } & 4 & 5,13 \% \\ \text { Caso encerrado*** } & 2 & 2,56 \% \\ \text { Óbito } & 2 & 2,56 \%\end{array}$

*A variável admite mais de uma alternativa. ** Pescoço, tórax anterior, tórax posterior, abdome. *** Caso dado como encerrado pela supervisão do CCI (sem dados elucidados).

Fonte: Autoras.

Em relação às características clínicas, nota-se que hiperemia, o eritema, o edema e a dor foram as alterações locais mais comuns entre os casos avaliados, sendo que cada uma foi observada em mais de $35 \%$ deles, enquanto que em relação as alterações sistêmicas, destacou-se o vômito, identificado em 32,05\% dos casos (Tabela 2).

O envenenamento, a partir das características clínicas do paciente, pode ser classificado em leve, moderado e grave. No estudo observou-se que a maior parte dos casos foi classificada como leve $(65,38 \%)$ e $89,74 \%$ evoluiu com alta hospitalar (Tabela 2).

Em relação aos sinais vitais e resultados dos exames, que estão dispostos na Figura 1 e Figura 2, respectivamente. Cabe destacar que esta análise foi realizada apenas para os casos classificados como moderado e grave. Nestas, as distribuições de frequências foram feitas considerando três possíveis resultados: alterado, representada na parte esquerda da figura; sem informação, representada ao centro e sem alteração, representada no lado direito.

Figura 1. Distribuição de frequências dos resultados dos sinais vitais/HGT/ECG das crianças e adolescentes com escorpionismo grave e moderado, de janeiro de 2014 a dezembro de 2018. Paraná, Brasil, 2019.

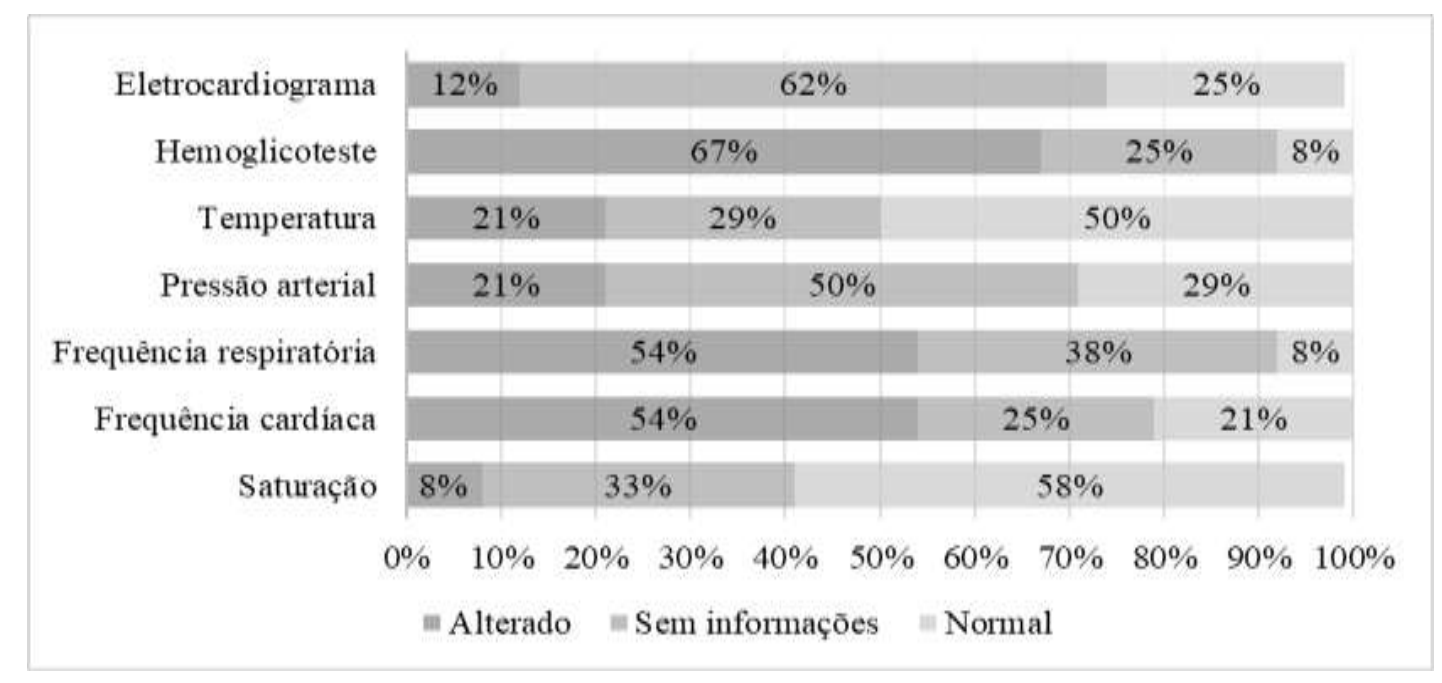

Fonte: Autoras.

Entre os pacientes classificados com envenenamento moderado (17) ou grave (7), notou-se que, para a maior parte dos dados, predomina-se resultados não informados. Porém, das poucas informações obtidas destacou-se alterações significativas para HGT $(67 \%)$, frequência cardíaca $(54 \%)$ e freqüência respiratória (54\%). Já a saturação (58\%) e a temperatura $(50 \%)$ tiveram grande parte dos resultados não alterados, como mostrou a Figura 1. Na figura 2 podemos observar a distribuição de frequências dos resultados dos exames complementares das crianças e adolescentes com escorpionimo grave e moderado. 
Research, Society and Development, v. 10, n. 1, e54610112079, 2021

(CC BY 4.0) | ISSN 2525-3409 | DOI: http://dx.doi.org/10.33448/rsd-v10i1.12079

Figura 2. Distribuição de frequências dos resultados dos exames complementares das crianças e adolescentes com escorpionimo grave e moderado por escorpião, de janeiro de 2014 a dezembro de 2018. Paraná, Brasil, 2019.

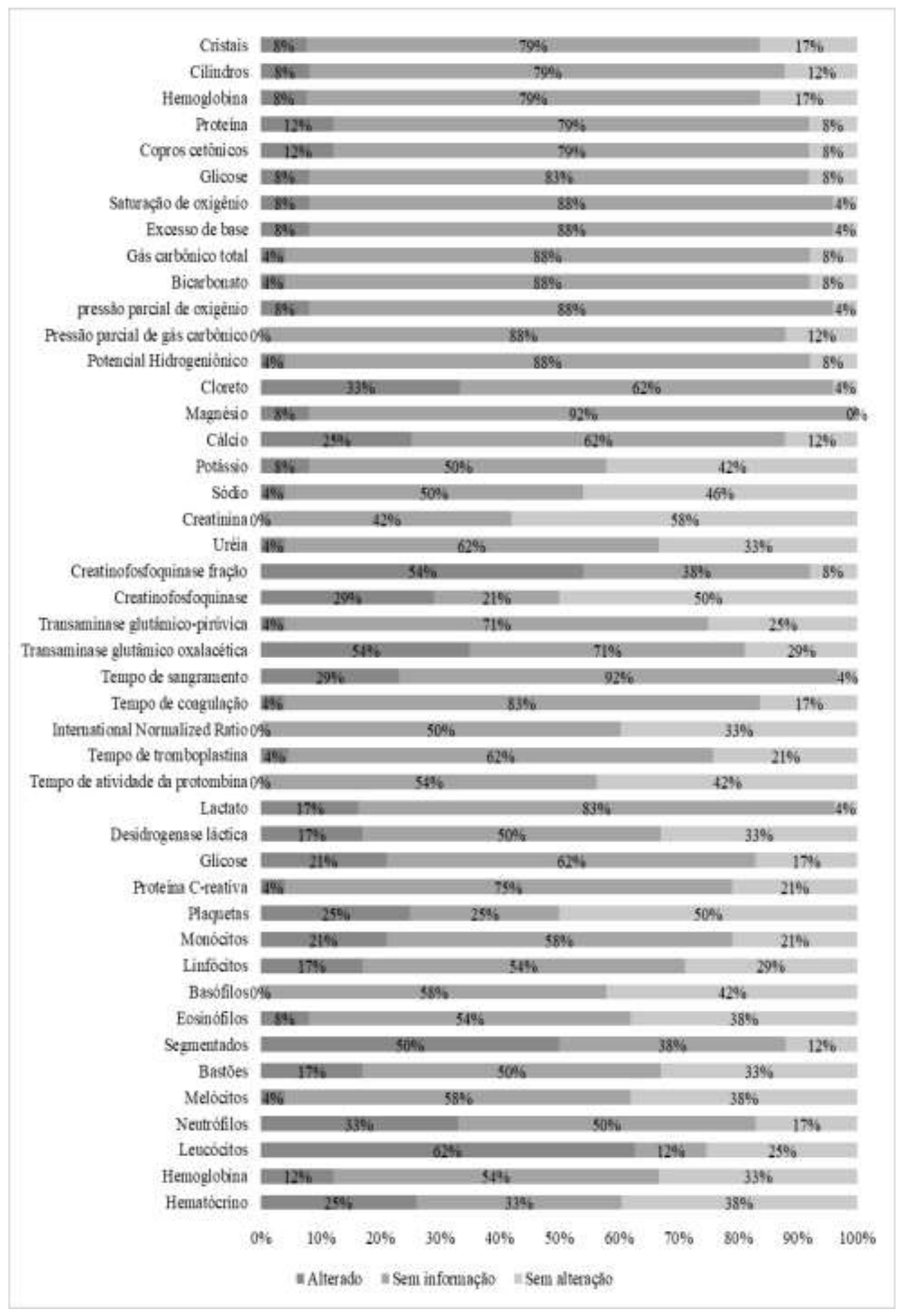

Fonte: Autoras. 
Para os resultados dos exames laboratoriais podemos destacar uma maior porcentagem nas alterações dos leucócitos (62\%), segmentados (50\%) e CKMB (54\%), ao passo que para as plaquetas (50\%), CPK (50\%) e creatinina (58\%), as alterações não foram observadas em grande parte, considerando o exposto na Figura 2. A Figura 3 apresenta o comportamento temporal da série mensal de ocorrências dos acidentes escorpiônicos.

Figura 3. Série mensal do número de ocorrências de acidentes escorpiônicos entre janeiro de 2014 e dezembro de 2018 . Paraná, Brasil, 2019.

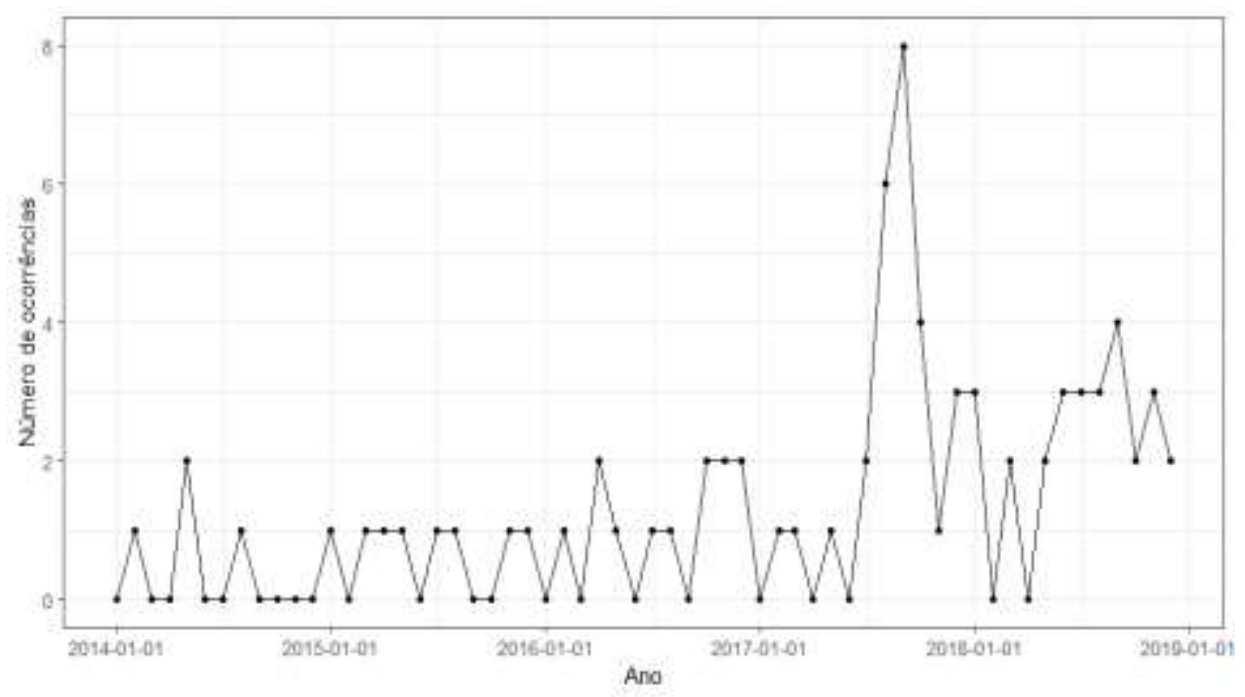

Fonte: Autoras.

Observa-se na Figura 3, de um modo geral, um crescimento ao longo do período avaliado, sendo que os anos de 2017 e 2018 apresentaram quase 70\% das ocorrências observadas. Destaca-se ainda um pico ocorrido em agosto e setembro de 2017, meses nos quais houve o registro de seis e oito ocorrências, respectivamente. O terceiro trimestre (julho a setembro), independente do ano, caracterizou-se como o período com maior número de ocorrências, quase $40 \%$ do total, ao passo que o primeiro (janeiro a março) e segundo (abril a junho) trimestre correspondem a apenas $14,10 \%$ e 16,67\% dos registros, respectivamente.

De acordo com os resultados da aplicação do teste não paramétrico de Mann-Kendall a série mensal de ocorrências de acidentes escorpiônicos mostrou que há evidências amostrais suficientes de existência de tendência significativa, ao nível de $5 \%$ de significância (valor p de 0,036 ), indicando um aumento no número de ocorrências ao longo do período.

\section{Discussão}

O escorpionismo é um problema de saúde pública com risco de morte em muitos lugares do mundo, e a faixa etária que tem maior probabilidade de desenvolver complicações e condições fatais são as crianças e os idosos (Furtado et al., 2020).

Neste estudo conseguimos mostrar que a faixa etária mais acometida foi a de até cinco anos com quase que a metade de todos os pacientes estudados. Dados estes que corroboram com a literatura (Gomes et al., 2020), onde alguns autores descrevem que essa idade pouco se restringe a berços e carrinhos e são altamente ativas (Santos et al., 2016), acabando por explorar lugares onde o animal costuma se abrigar (Sathiadas et al., 2017; Bhamkar et al., 2016). Além disso é uma das faixas etárias que está diretamente associada aos quadros de maior gravidade pois demonstra maior vulnerabilidade à toxína escorpiônica e maior susceptibilidade às complicações (Carmo et al., 2019). Houve também a predominância do sexo 
masculino, o que pode ser confirmado em vários outros estudos (Bhamkar et al., 2016; Santos et al., 2016; Sathiadas et al., 2017; Saad et al., 2017; Carmo et al., 2019; Torrez et al.,2019; Gomes et al., 2020).

Observou-se ainda que a maioria dos pacientes residia na zona urbana e que o acidente ocorreu em sua própria residência. Esses dados corroboram com outro estudo, o qual ainda revela que os acidentes ocorridos em locais distantes de serviços de saúde, como na zona rural, estão diretamente ligados a um pior estado clínico, pois levam maior tempo para receberem a terapêutica adequada (Carmo et al., 2019).

Em dois terços dos casos avaliados o agente envolvido no acidente foi o Tityus Serrulatus que, de acordo com a literatura (Feitosa et al., 2020; Ministério da Saúde, 2019b), é a espécie de maior importância médica devido o alto grau de complicações causadas aos seres humanos quando há inoculação de sua toxína. De acordo com o Sistema de Informação de Agravos de Notificação (SINAN), houve um aumento no número de casos registrados entre 2013 a 2017 nas regiões Norte, Centro-Oeste e Sul, onde Tityus Serrulatus não ocorria ou raramente ocorria (Ministério da Saúde, 2018), fato este que explica o aumento da incidência de acidentes com esta espécie na região estudada. É importante considerar também que a urbanização acelerada nas últimas décadas, sem infraestrutura básica adequada, tem proporcionado a proliferação destes animais, sempre destacando que essa espécie é partogenética, ou seja, a fêmea pode se procriar sem ser fertilizada pelo macho (Ministério da Saúde, 2009).

Ressalta-se que a maioria dos atendimentos foi realizado em até duas horas após a picada, o que influenciou na gravidade dos sintomas no momento da admissão, ou seja, pacientes atendidos após duas horas do acidente apresentaram um risco maior de evolução desfavorável (Chtara et al., 2015). Dados estes que reforçam o ocorrido neste estudo, onde a média de atendimento maior foi em tempo inferior a uma hora, entre o acidente e o primeiro atendimento. Podemos destacar também que a chance de uma criança evoluir à óbito aumenta em nove por cento a cada hora de atraso em um atendimento e a mesma chance passa de $13 \%$ a cada ano diminuído na idade desta criança, portanto, podemos reforçar que quanto menor o tempo decorrido entre o acidente e o atendimento é essencial para a melhora do paciente (Carmo et al., 2019).

As regiões anatômicas da picada mais frequentes observadas nos 78 pacientes estudados foram: os membros inferiores e superiores, respectivamente, o que se iguala a alguns dos estudos já realizados que identificam as extremidades como as mais atingidas pela picada, provavelmente por serem as estruturas que ficam mais em evidência (Bhamkar et al., 2016; Santos et al., 2016; Sathiadas et al., 2017; Carmo et al., 2019; Gomes et al., 2020, Furtado et al., 2020).

Quanto às características clínicas, as mesmas foram encontradas em outros estudos, com destaque para a dor local com hiperemia nos sinais clínicos (Bhamkar et al., 2016; Santos et al., 2016; Sathiadas et al., 2017; Carmo et al., 2019; Gomes et al., 2020, Furtado et al., 2020; Saad et al., 2017; Torrez et al., 2019; Ministério da Saúde, 2018; Chtara et al., 2015; Monteiro et al., 2019).

Lembrando que a dor na criança pode levar à quadros de náusea, agitação e taquicardia que desaparecerão após o tratamento local, ou não, por isso, em todos os casos recomenda-se a observação de, no mínimo, seis horas para melhor acompanhamento da evolução (Saad et al., 2017). Já pacientes mais graves podem ter sintomas como vômito, agitação, taquipnéia, alteração na pressão arterial, sudorese, alteração na freqüência cardíaca e até mesmo sintomas de insuficiência cardíaca congestiva aguda em consequência do aumento da resistência vascular e do edema pulmonar, o que seria indicação de soroterapia na conduta (Ministério da Saúde, 2009).

Em relação à evolução dos casos, quase dois terços do total foram classificados como leves. A maior parte recebeu alta e dois pacientes foram a óbito. Alguns estudos evidenciaram que a maioria dos casos é classificada como leve, e grande parte evolui para cura (Bhamkar et al., 2016; Santos et al., 2016; Sathiadas et al., 2017; Carmo et al., 2019; Gomes et al., 2020, Furtado et al., 2020), o que corrobora com este estudo que demonstra uma melhora clínica considerável da população estudada. 
Para as análises realizadas nas fichas dos pacientes classificados como moderados e graves destacamos que, o critério de escolha foi utilizado devido a identificação de que a maioria dos casos leves não tiveram registros de alterações em sinais vitais e exames complementares. Sendo assim, nesses pacientes classificados com escorpionísmo moderado ou grave. Notou-se pouca alteração dos exames laboratoriais, com exceção do HGT, leucócitos, segmentados e CKMB. Ressalta-se que a picada de escorpião leva a liberação de hormônios que suprimem a secreção de insulina e/ou causam resistência à mesma, o que acaba por ocasionar a hiperglicemia (aumento do HGT), resultando em um mal prognóstico, principalmente em crianças (Triches, 2017). Um trabalho experimental revelou que, houve alterações significativas nos exames laboratoriais aos sujeitos expostos a toxina de escorpião ${ }^{22}$, o que também reforça alguns dados expostos neste trabalho.

A série mensal de ocorrências dos acidentes apresentou um comportamento crescente ao longo do período avaliado, com pico ocorrido em agosto e setembro de 2017, sendo que julho, agosto e setembro de todos os anos foi o período com maior número de ocorrências. Os achados vêm de acordo com a literatura encontrada ao qual mostra que a maioria dos acidentes ocorre durante todo o ano, porém com uma tendência a aumentar durante a estação chuvosa, independente das regiões estudadas, o que acaba por resultar na saída dos escorpiões de seus habitats a procura de abrigo e alimento (Gomes et al., 2016).

É importante salientar que a tendência crescente de acidentes com escorpião encontrada na pesquisa pode estar relacionada ao aumento das notificações dos casos, o que se deve ao maior conhecimento da população sobre o animal com o passar dos anos. O manual do Ministério da Saúde demonstra o aumento das taxas desses acidentes e dispõe sobre os cuidados para prevenção do mesmo para que os serviços de saúde possam fazer as orientações à população (Ministério da Saúde, 2009).

No que se refere às limitações do estudo, destaca-se a pouca diversidade de estudos nacionais e internacionais atuais sobre o tema envolvendo o público pediátrico, o que comprometeu a comparabilidade dos dados, e também, a utilização de dados secundários, os quais podem apresentar subregistros.

\section{Conclusão}

Evidenciou-se uma incidência maior em crianças menores de cinco anos, que é a faixa etária mais propensa a complicações, e também uma crescente no número de casos ao longo do período estudado, o que configura um sério problema de saúde pública no município estudado e região.

Estes resultados indicam a necessidade de novos estudos epidemiológicos envolvendo o público pediátrico buscando investigar as características destes acidentes a fim de contribuir para o melhor delineamento das ações de prevenção e promoção da saúde. Além disso, estes dados mostram a importância de campanhas educativas pensando na educação sanitária da comunidade, principalmente em relação ao cuidado com as crianças, e treinamento específico para profissionais de saúde, para que os mesmos possam reconhecer precocemente a gravidade destes acidentes.

Sugere-se ainda o desenvolvimento de mais pesquisas acerca da temática com um número amostral maior, visto os benefícios que a compreensão deste fenômeno pode trazer aos cuidados de crianças picadas por escorpião.

\section{Referências}

Araújo, K. A. M., Tavares, A. V., Marques, M. R. de V., Vieira, A. A., \& Leite, R. de S. (2017). Epidemiological study of scorpion stings in the Rio Grande do Norte State, Northeastern Brazil. Revista do Instituto de Medicina Tropical de São Paulo, 59: e58.

Bhamkar, R., Seth, B., \& Setia, M. S. (2016). Profile and Risk Factor Analysis of Unintentional Injuries in Children. Indian Journal of Pediatrics, 83: 111420 .

Carmo, E. A., Nery, A. A., Paula, R. P., Rios, M. A., \& Casotti, C. A. (2019). Fatores associados à gravidade do envenenamento por escorpiões. Texto \& Contexto Enfermagem, 28: e20170561. 
Chtara, K., Bahloul, M., Turki, O., Baccouche, N., Regaieg, K., Chokri, B., et al. (2015). Incidence and impact outcome of hyperglycaemia in severe scorpion envenomed children requiring intensive care admission. Intensive Care Medicine, 41(10): 1871-2.

Feitosa, A. M., Camplesi, A. C., Pinheiro, J. A., Mathias, L. A., \& Belo, M. A. (2020). Incidence of scorpiotic accidents in the municipality of ilha solteira-sp. Ars veterinária, 36(2): 88-97.

Furtado, A. A., Silva, A. D., Silva-Júnior, A. A., \& Pedrosa, M. F. F. (2020). Biology, venom composition, and scorpionism induced by brazilian scorpion Tityus stigmurus (Thorell, 1876) (Scorpiones: Buthidae): A mini-review. Toxicon, 185: 36-45.

Gomes, J. V., Fé, N. F., Santos, H. L. R., Jung, B., Bisneto-Ferreira, P., Sachett, A., et al. (2020). Clinical profile of confirmed scorpion stings in a referral center in Manaus, Western Brazilian Amazon. Toxicon, 187: 245-54.

Guimarães, P. T. C., Pinto, M. C. L., \& Melo, M. M. (2011). Hematological and clinical profiles of mice submitted to experimental poisoning with Tityus fasciolatus venom. Arquivo Brasileiro de Medicina Veterinária e Zootecnia, 63(6): 1382-90.

Lisboa, N. S., Boere, V., Neves, F. M. (2020). Scorpionism in the far south of Bahia, Brazil, 2010-2017: case profile and factors associated with severity. Epidemiologia e Serviços de Saúde, 29(2): e2019345.

Machiwal, D., \& Jha, M. K. (2012). Hydrologic time series analysis: theory and practice. New Delhi: Springer.

Ministério da Saúde. (2018). Secretaria de Vigilância em Saúde (SVS). Sistema de Informação de Agravos de Notificação (SINAN). Saúde de A a Z. Acidentes por animais peçonhentos. https://antigo.saude.gov.br/saude-de-a-z/acidentes-por-animais-peconhentos

Ministério da Saúde (2019a). Secretaria de Vigilância em Saúde. Acidentes de trabalho por animais peçonhentos entre trabalhadores do campo, floresta e águas, Brasil 2007 a 2017. Boletim Epidemiológico, 50(11): 1-14.

Ministério da Saúde (2009). Secretaria de Vigilância em Saúde. Departamento de Vigilância Epidemiológica. Manual de controle de escorpiões. http://bvsms.saude.gov.br/bvs/publicacoes/manual_controle_escorpioes.pdf

Ministério da Saúde (2019b). Secretaria de Vigilância em Saúde. Guia de Vigilância em Saúde. Brasília: Ministério da Saúde. https://bvsms.saude.gov.br/bvs/publicacoes/guia_vigilancia_saude_3ed.pdf

Ministério da Saúde (2021). Sistema de Informação de Agravos de Notificação. Acidente por animais peçonhentos. Notificações registradas no sistema de informação de agravos de notificação. http://tabnet.datasus.gov.br/cgi/tabcgi.exe?sinannet/cnv/animaispr.def.

Monteiro, W. M., Gomes, J., Fé, N., Silva, I. M., Lacerda, M., Alencar, A., et al. (2019). Perspectives and recommendations towards evidence-based health care for scorpion sting envenoming in the Brazilian Amazon: A comprehensive review. Toxicon, 169: 68-80.

Pereira, A. S., Shitsuka, D. M., Parreira, F. J., \& Shitsuka, R. (2018). Metodologia da pesquisa científica: Universidade Federal de Santa Maria.

R Development Core Team (2015). R: a language and environment for statistical computing. R Foundation for Statistical Computing.Vienna, Austria.

Saad, K., El-Hamed, M. A. A., Abo-Elela, M. G. M., Ahamed, A. E., Abdel-Baseer, K. A., Aboul-Khair, M. D., et al. (2017). Neurologic Complications in Children with Scorpionism: A Retrospective Study in Upper Egypt. Journal of Child Neurology, 32(6):537-42.

Santos, M. S. V., Silva, C. G. L., Neto-Silva, B., Júnior-Grangeiro, C. R. P., Lopes, V. H. G., Júnior-Teixeira, A. G., et al. (2016). Clinical and epidemiological aspects of scorpionism in the world: a systematic review. Wilderness \& Environmental Medicine, 27(4): 504-18.

Sathiadas, M. G., Quinters, V., Ketheeswaran, N., Mohamed, A. A., \& Karunya, V. (2017). Epidemiology, Clinical Profile e Outcome of Stings and Bites in Children Admitted to a Tertiary Care Hospital. Archives de Pédiatrie, 3(2): JPED-115.

Torrez, P. P. Q., Dourado, F. S., Bertani, R., Cupo, P., \& França, F. O. S. (2019). Scorpionism in Brazil: exponential growth of accidents and deaths from scorpion stings. Revista do Instituto de Medicina Tropical de São Paulo, 52: e20180350.

Triches, C. M. F. (2017). Predição e caracterização de um epitopo conformacional da beta-neurotoxina TSI do escorpião Tityus serrulatas [Dissertação de Mestrado]: Universidade do Extremo Sul Catarinense. 